\title{
Performance Evaluation of the Quantitative Point-of- Care LumiraDx D-Dimer Test
}

\author{
Jayne E. Ellis · Thomas W. Johnston · David Craig · Anita Scribner • \\ William Simon · Judith Kirstein
}

Received: July 19, 2021 / Accepted: September 8, 2021 / Published online: October 7, 2021

(C) The Author(s) 2021

\begin{abstract}
Introduction: Fibrin degradation product Ddimer can be a valuable indicator for venous thromboembolism (VTE). The use of D-dimer testing in primary care settings can be limited by restricted access to laboratory services. This performance evaluation compares a quantitative, point-of-care (POC) D-dimer assay (LumiraDx D-Dimer Test) with a reference laboratorybased D-dimer assay.

Methods: Plasma samples from patients presenting to secondary care in the UK, USA, and Germany were analyzed centrally using the LumiraDx D-Dimer Test and the reference test (bioMérieux VIDAS D-Dimer Exclusion II immunoassay). Method comparison used Passing-Bablok regression analysis with pre-specified equivalence criteria of $r \geq 0.9$ and slope of
\end{abstract}

J. E. Ellis $(\bowtie) \cdot$ T. W. Johnston · D. Craig

LumiraDx, Stirling, UK

e-mail: jayne.ellis@lumiradx.com

A. Scribner

Diagnostic Clinic of Longview, Longview, TX, USA

W. Simon

New Medical Healthcare, Wichita, KS, USA

J. Kirstein

Velocity Clinical Research, Banning, CA, USA

J. E. Ellis

LumiraDx Ltd, 3 More London Riverside, London

SE1 2AQ, UK
0.9-1.1. The NOVEL-3 study (NCT04375982) compared equivalency of fingerstick, venous blood (VB), and plasma samples from the same patient, tested at US primary care clinics next to the patient using the POC LumiraDx D-Dimer device. Measurements obtained from fingerstick and VB samples were compared with results from plasma samples, using Deming regression. The healthy reference range was determined using plasma samples of healthy volunteers, collected by commercial suppliers in Germany and the USA, which were analyzed centrally using the LumiraDx D-Dimer Test and the reference test.

Results: The LumiraDx D-Dimer Test demonstrated agreement with the bioMérieux VIDAS D-Dimer Exclusion II immunoassay for plasma samples $(r=0.923$, slope of $1.016, n=1767)$. There was good agreement between fingerstick/ VB samples and plasma samples ( $r=0.980-0.986, n=93)$ measured using the LumiraDx D-Dimer Test. Overall error rates were $1.8 \%$. The healthy reference range $90 \%$ percentile for D-dimer was calculated as $533 \mu \mathrm{g} / \mathrm{l}$ fibrinogen equivalent units (FEU).

Conclusions: The quantitative LumiraDx DDimer Test is easy to use and can accurately measure D-dimer levels in a range of blood sample types, including fingerstick samples, which could improve assessment of VTE cases in community and hospital near-patient settings. 
Keywords: Deep vein thrombosis; D-dimer; Point-of-care testing; Primary care; Pulmonary embolism; Quantitative D-dimer testing; Venous thromboembolism

\section{Key Summary Points}

A quantitative, point-of-care (POC)

D-dimer test could improve the

effectiveness of the established diagnosis pathway of venous thromboembolism (VTE), thereby reducing the current rate of unnecessary referrals to secondary care along with associated cost and improve patient experience.

The primary aim of this study was to assess the performance of the new quantitative point of care LumiraDx D-Dimer Test for accuracy, precision, and measurement repeatability across several sample types, including fingerstick capillary blood, compared to established, laboratory-based analysis.

The quantitative LumiraDx D-Dimer Test was able to accurately measure D-dimer levels using samples across the range 60 $\mu \mathrm{g} / \mathrm{l}$ and $4515 \mu \mathrm{g} / \mathrm{l}$ fibrinogen equivalent units (FEU), with a low error rate across a range of blood sample types.

The D-dimer test was shown to be easy to use by users in POC settings.

The study results show that this novel quantitative POC test provides accurate results at the point of care when patients are presenting with symptoms of VTE and a quantitative D-dimer test is quickly needed. When utilized in line with the diagnostic guidance, this could facilitate more accurate referrals for imaging diagnostics of VTE in settings such as primary care, where most patients initially present.

\section{INTRODUCTION}

Venous thromboembolism (VTE) is a collective term for deep vein thrombosis (DVT) and pulmonary embolism (PE), which represents a significant cause of mortality and disability, affecting 1-2 per 1000 people of European ancestry annually [1]. The high level of VTEassociated morbidity is at least partly due to symptoms of VTE being diverse, making its diagnosis challenging.

D-dimer is a fibrin degradation product resulting from the degradation of thrombi by fibrinolysis. During coagulation, fibrinogen is converted by thrombin to fibrin monomers, which are subsequently cross-linked by factor XIIIa. When fibrin is degraded, the final step, consisting of plasmin cleavage, results in the formation of D-dimer and factor E [2]. Low levels of D-dimer can be found in the whole blood and plasma of healthy individuals, but are elevated in patients with thrombosis, making it a valuable indicator for VTE [2]. The initial diagnosis pathway for DVT generally begins in the primary care setting, whereas the diagnosis pathway for PE most often starts in the emergency department with patients presenting with chest pain. Patients with suspected VTE are subsequently referred to hospital departments with appropriate blood analysis and imaging capabilities. However, many of the patients assessed in the hospital do not have VTE [2]. In fact, the typical ratio of imaging-confirmed VTE versus a negative diagnosis is 1:6 [3]. Conversely, there are patients who require further diagnostic tests but are not referred because they present with non-specific symptoms; such patients are at risk of receiving a delayed diagnosis, which could consequently lead to adverse outcomes [4, 5].

Excessive referrals for imaging tests carry concomitant cost. The associated cost reduction of successfully implementing a VTE diagnostic strategy involving initial clinical assessment versus imaging alone was found to be $38 \%$ for patients with suspected PE and $24 \%$ for suspected DVT. Assuming the proportion of suspected PE and DVT patients to be $30 \%$ and $70 \%$, respectively, the weighted average cost reduction per suspected VTE patient was 32\% [6]. 
Efforts to reduce the rate of unnecessary imaging referrals have led to the implementation of the Wells' clinical scoring criteria for DVT and PE in settings where patients with VTE commonly present [7]. If the resulting score indicates that VTE is likely, the patient is referred for a diagnostic imaging test and D-dimer assay confirmation [7]. Recent UK National Institute for Health and Care Excellence (NICE) guidelines also recommend performing a confirmatory D-dimer test in patients whose clinical assessment score indicates that VTE is unlikely. Point-of-care (POC) tests for D-dimer, preferably those able to give a quantitative result, are recommended if laboratory facilities are not immediately available. Quantitative tests are preferred owing to the recognition that an ageadjusted D-dimer threshold may be advisable for patients over 50 years of age [8]. Generally, used in combination with a risk assessment, a Ddimer concentration of $<500 \mu \mathrm{g} / \mathrm{l}$ is used to exclude VTE, whereas assay results of $>500 \mu \mathrm{g} / \mathrm{l}$ indicate the need for a diagnostic imaging test, even if clinical assessment alone suggests that VTE is unlikely [9]. However, it must be noted that commercially available D-dimer assays use different antibodies and have different clinical cut-offs, which must be established from clinical studies. Therefore, cut-off thresholds are assay-specific and not always $500 \mu \mathrm{g} / \mathrm{l}$ [10]. To date, the established method for the detection of D-dimer is the enzyme-linked immunosorbent assay, which is generally performed in a central clinical laboratory [11]. A number of qualitative and quantitative POC tests have been developed for the evaluation of D-dimer concentrations, which utilize a variety of methodologies [11]. The LumiraDx device is a microfluidic immunoassay using fluorescence detection. A small sample of blood $(15 \mu \mathrm{l})$ is applied to the test strip by way of direct application (DA) in the case of fingerstick DA, or use of a lithium heparin anticoagulated transfer tube (TT) for fingerstick blood. Plasma and venous whole blood (citrated) are applied to the strip by pipette $(20 \mu \mathrm{l})$. The test strip contains magnetic particles as well as fluorescent latex particles in the pre-applied reagent. When Ddimer is present, the different antibody-bound particles form a fluorescent, magnetic D-dimer complex. This complex is kept in place by a magnet below the optical block, which allows the fluorescence, which is proportional to the captured D-dimer, to be measured.

The NICE recommendation for POC testing for D-dimer in the primary care setting has the potential to reduce the number of unnecessary hospital referrals, thereby improving patient experience on the diagnosis pathway and relieving pressure on healthcare resources $[12,13]$. Indeed, prospective management studies have shown that even qualitative POC Ddimer tests can rule out a large proportion of patients who do not need a referral (up to 49\%) $[12,14,15]$, with low false-negative rates (1.4-2\%) [12]. Moreover, the sensitivity and specificity of a combined clinical score and qualitative POC D-dimer test were comparable to the laboratory-based quantitative test [15]. An assessment of POC testing for D-dimer in the diagnosis of DVT found it to be cost-effective [16]. Moreover, the convenience of having the results available at POC when the patient is present allows for more efficient use of resources overall [16]. The limited evidence available to date suggests that POC D-dimer assays can improve the effectiveness of the diagnosis pathway of VTE. An advantage of a quantitative POC assay over laboratory testing is that ageadjusted cut-off values can be implemented [12].

The primary aim of this performance evaluation was to assess the performance of the new quantitative LumiraDx D-Dimer Test (LumiraDx, Stirling, UK) for accuracy, precision, and measurement repeatability across several sample types (fingerstick blood, venous blood [VB], and plasma), and to determine usability and error rates of the assay.

\section{METHODS}

\section{Studies}

The LumiraDx D-Dimer Test was evaluated in three separate studies. The first was a method comparison study of the LumiraDx device and the established bioMérieux VIDAS D-Dimer Exclusion II immunoassay (bioMérieux, Marcy 
l'Etoile, France), which used venous and capillary blood samples collected as part of the NOVEL study conducted in UK hospitals [17], and plasma samples from commercial suppliers from the USA and Germany.

The second was the NOVEL-3 matrix comparison study (NCT04375982), which assessed the equivalence of different testing modalities and was conducted across multiple POC testing sites in the USA.

The third study aimed to determine the healthy reference range for the LumiraDx DDimer Test. Samples were collected from healthy subjects by commercial suppliers who recruited participants in the USA (Logical Biological, Sandwich, UK, and Innovative Research Inc., Minneapolis, MN, USA) and Germany (TCS Biosciences Ltd, Buckingham, UK).

\section{Study Participants}

Participants for all sub-studies were at least 18 years of age and willing to provide written informed consent for laboratory testing. Participants in the NOVEL study were invited to take part when presenting at a participating hospital with symptoms indicative of heart failure, acute coronary syndrome, kidney failure, thromboembolic events, or inflammatory disorders. However, vulnerable populations deemed inappropriate for the study by the principal investigators of the participating sites were excluded.

Participants for the NOVEL-3 study were invited to take part when presenting to a healthcare provider for any reason, though the following conditions were of particular interest: VTE, patients seeking medical attention with symptoms of non-COVID-19 respiratory tract infection (upper or lower), acute myocardial infarction/unstable angina, patients on anti-inflammatory medication (current) or any regular medication for a chronic condition (other than simple painkillers or an inhaler), atrial fibrillation, diabetes mellitus (all types except 'pre-diabetes'), heart failure, hypertension ( $>150$ / $90 \mathrm{mmHg}$ ), infection (significant, current, or within 3 months), peripheral arterial disease, surgery (within 6 weeks), childbirth (within
8 weeks), significant trauma, burns (within 4 weeks), pregnancy (confirmed or suspected), acute upper gastrointestinal hemorrhage or other significant recent hemorrhage. Patients were excluded from the NOVEL-3 study if they were currently receiving or had received an experimental biologic or drug, including treatment or therapy within the 30 days prior to the study visit, if they had skin lesions or conditions that would preclude a fingerstick and/or a VB draw, end-stage renal failure on hemodialysis, or life expectancy documented as less than 30 days, if they were classified as hemodynamically unstable (e.g., cardiogenic shock) and if they had been taking anticoagulant therapy (direct oral anticoagulants, warfarin, heparins, etc.) within the last 30 days.

Study participants for the healthy reference range analysis were required to be in good health and were excluded if they had any current or prior incidence of: acute myocardial infarction/unstable angina, peripheral arterial disease, superficial thrombophlebitis, atrial fibrillation, arterial or venous thromboembolism (under active treatment), heart failure, aortic dissection/aneurysm, hypertension (>150/ $90 \mathrm{mmHg}$ ), disseminated intravascular coagulation, sickle cell disease/hemolysis, infection (significant, current or within 3 months), acute upper gastrointestinal hemorrhage, other significant recent hemorrhage, diabetes mellitus (all types except 'pre-diabetes'), connective tissue disease (systemic lupus erythematosus, systemic sclerosis), rheumatoid arthritis, malignancy (active or currently treated), chronic kidney disease (estimated glomerular filtration rate $<60 \mathrm{ml} / \mathrm{min}$ ), severe liver disease (cirrhosis/ascites), pregnancy (confirmed or suspected), childbirth (within 8 weeks), stroke (ever), significant trauma, burns (within 4 weeks), surgery (within 6 weeks), immobilization (prolonged), syncope (recent, hospitalized or admitted to the accidents and emergency department), hyperlipidemia/hypertriglyceridemia, or hyperbilirubinemia. Patients who were receiving the following treatments were also excluded: anticoagulant therapy, fibrinolytic therapy, hemodialysis, anti-inflammatory medication, combined oral contraceptive (medium- or high-dose) or any regular 
medication for a chronic condition (other than simple painkillers or an inhaler).

\section{Study Design}

The primary objective of this performance evaluation was to show equivalence between the quantitative LumiraDx D-Dimer Test using different sample types and the established, quantitative laboratory-based bioMérieux VIDAS D-Dimer Exclusion II immunoassay using plasma samples. Samples for the NOVEL study, an ongoing prospective blood collection study, were collected at multiple hospital sites in Scotland, UK. Samples of whole sodium-citrated VB were collected from eligible patients using standard venepuncture technique performed by trained staff and processed to plasma, before being sent to LumiraDx (LumiraDx UK Ltd, Stirling, UK) for analysis. Plasma samples were stored at $-70^{\circ} \mathrm{C}$ for up to 2 years. Additional plasma samples were provided by commercial suppliers (Logical Biological, Innovative Research, Inc., TCS Biosciences Ltd). Plasma samples were analyzed at the sponsor site using three LumiraDx D-Dimer Test strip lots and the bioMérieux VIDAS D-Dimer Exclusion II immunoassay. Plasma samples were tested in replicates of two for each sample and test strip lot, and in accordance with the manufacturer's instructions [18, 19]. The NOVEL study complied with the Declaration of Helsinki (2013) and was approved by West of Scotland REC 3 (REC number 15/WS/0176 and IRAS ID: 179093). Commercial samples were obtained under local protocol and ethical approval.

For the NOVEL-3 study, samples were used to perform matrix comparisons and precision at POC in subjects recruited in four primary healthcare centers in the USA. Patients were recruited and staff collected from each participant two sample tubes, containing sodium citrate, of VB using standard venepuncture technique, and four fingerstick samples of capillary blood using a fingerstick lancet. Samples were taken in duplicate to allow for replicate testing of each sample method. Fingerstick samples were applied to the LumiraDx test strips as whole blood DA or using a TT. The fingerstick samples were analyzed at POC using the LumiraDx D-Dimer Test following the manufacturer's instructions for use [19]. The test procedure, from inserting the test strip into the device to collecting and applying the sample to the test strip, took approximately $1 \mathrm{~min}$, with a further $6 \mathrm{~min}$ required for running the test and obtaining results. During the study, one of the VB tubes was used immediately with the POC LumiraDx D-Dimer Test, and the remainder of this tube and the second tube were processed to plasma and transferred to the sponsor site for reference testing using the bioMérieux VIDAS DDimer Exclusion II immunoassay. Unused samples were stored at $-70^{\circ} \mathrm{C}$ and tested within 1 month, if required. The number and type of error codes observed were recorded, and usability was assessed using a questionnaire. The study protocol for NOVEL-3 complied with the Declaration of Helsinki (2013) and IRB approval was obtained from WCG ASPIRE IRB (ID: 14-1290899-1).

For the healthy reference range, VB samples in sodium citrate were obtained from a healthy population by standard venepuncture technique performed by trained staff. Suppliers processed the blood to plasma, froze aliquots, and shipped the samples to LumiraDx, Stirling, $\mathrm{UK}$, for testing. Samples were stored at $-70^{\circ} \mathrm{C}$ for up to 6 months before testing. The bioMérieux VIDAS D-Dimer Exclusion II immunoassay was used as the reference method, consisting of two replicates of each sample. Samples were tested using three LumiraDx D-Dimer Test strip lots in two replicates, resulting in six measurements for each sample. Assays were performed in accordance with the manufacturers' instructions $[18,19]$. Local ethics committee approval was obtained as required by commercial sample providers.

\section{Operator Usability Assessment}

During the prospective fingerstick study (NOVEL 3), the LumiraDx D-Dimer Test was assessed for the ease of use for operators without any prior training at the POC settings. The usability was assessed with nine untrained users 
across four test sites in the USA, testing a total of 120 subjects in total, resulting in 1101 tests. Usability was determined by analysis of errors obtained during the study, ease of recovery from errors, and study questionnaire responses. The Intended Use Operator Questionnaire evaluated various metrics of test usability and safety. Each question was completed using a five-point Likert scale, ranging from $1=$ strongly disagree to $5=$ strongly agree (Fig. 3).

\section{Statistical Analysis}

Sample size was determined in line with Clinical and Laboratory Standards Institute (CLSI) guidelines (CLSI EP09C ED3:2018 [20] and CLSI EP28 A3C:2010 [21]). For the method comparison using samples from the NOVEL study, the average of the two replicates measured using the bioMérieux VIDAS D-Dimer Exclusion II immunoassay was used in the analysis. A Passing-Bablok regression analysis was used, with pre-specified criteria for equivalence of $r \geq 0.9$, a slope of 0.9-1.1 and an intercept between 200 and 200.

Deming regression was used to assess equivalence of sample types analyzed with the LumiraDx D-Dimer Test for the NOVEL-3 study samples.

Within the healthy reference range analysis, the average of the two replicates assessed using the bioMérieux VIDAS D-Dimer Exclusion II immunoassay was used as the reference. A nonparametric approach was used to determine the healthy reference range of the LumiraDx DDimer Test. Analyses were performed separately for each LumiraDx test strip lot tested and for the overall dataset to assess lot-to-lot strip variability.

A precision study was carried out in citrated venous plasma on a protocol based on CLSI EP5A3 [22]. For this, three D-dimer concentrations were each tested in two runs of two replicates per day, for 20 days. Intra-run, intra-day, interday, and total precision were assessed for a total of 80 samples (Table 1). The coefficient of variation (\% CV) was calculated for each analysis for each D-dimer concentration. A test with an analytical variability of $\leq 10 \%$ was considered to have high precision.

\section{RESULTS}

\section{Participant and Sample Characteristics}

Samples from a total of 327 participants enrolled in the NOVEL study from seven sites in Scotland, UK (Edinburgh Royal Infirmary, Edinburgh; University Hospital Wishaw, Wishaw; University Hospital Monkland, Airdrie; University Hospital Hairmyres, Glasgow; Queen Elizabeth University Hospital, Glasgow; Glasgow Royal Infirmary, Glasgow; Golden Jubilee National Hospital, Clydebank), together with samples from external suppliers sourced from Germany and the USA (Logical Biological; TCS Biosciences; Innovative Research) were used for the method comparison of the LumiraDx D-Dimer Test with the bioMérieux VIDAS D-Dimer Exclusion II immunoassay. The mean age of participants was 51 years (SD 20.5, range $18-98)$, and $41 \%$ were female. Most participants were presenting in hospital with suspected VTE, chest pain, or heart failure. Samples were collected between July 2019 and August 2020 .

A total of 100 participants were enrolled at four test sites in the USA (Rancho Paseo Medical Group, Banning, CA, USA; Centura Health Physician Group, Northglenn, CO, USA; New Medical Health Care, Wichita, KS, USA; Diagnostic Clinic of Longview, Longview, TX, USA) for the NOVEL-3 study, from April 2020 to June 2020. The mean age of enrolled participants was 50 years (SD 17; range 18-79), and 64\% were female. The most common diagnoses in the participant population were hypertension and diabetes.

Healthy reference range data were obtained from 127 participants enrolled in Germany and the USA from March to April 2020. The reference population consisted of 52 female and 75 male participants, with an age range of 18-50 years old (average age of 31.5 and 33.1 years for females and males, respectively). 


\section{Method Comparison of the LumiraDx and bioMérieux VIDAS Assays}

The accuracy of the LumiraDx D-Dimer Test was assessed by comparing results for plasma samples from 327 patients (range 60-4515 $\mu \mathrm{g} / \mathrm{l} \mathrm{FEU}$ [Fibrinogen Equivalent Units]) with those obtained using the bioMérieux VIDAS D-Dimer Exclusion II immunoassay $(n=1767)$. Although the Passing-Bablok analysis showed less agreement for samples greater than $1000 \mu \mathrm{g} / \mathrm{l}$, the analysis met the stated performance criteria ( $\mathrm{r}$ $\geq 0.9$, a slope of $0.9-1.1$ and an intercept between - 200 and 200) with $r=0.923$, a slope of 1.016 and an intercept of 21 (Fig. 1).

\section{Agreement Between Sample Types}

D-dimer values from the 100 plasma samples included in the analysis ranged from 55 to $3335 \mu \mathrm{g} / \mathrm{l}$ as assessed by the bioMérieux VIDAS D-Dimer Exclusion II immunoassay. Deming regression showed good agreement between the fingerstick and plasma results measured using the LumiraDx D-Dimer Test, with $r$ values ranging from 0.980 to 0.982 (Fig. 2). The VB results also showed a good correlation with the plasma results $(r=0.986)$. There was a noted under-recovery in $\mathrm{VB}$ results relative to the plasma results at higher D-dimer concentrations (Fig. 2).

The precision analysis of three different Ddimer concentrations showed a \%CV of $10.3 \pm 0.7$ for within-run and within-day precision as well as total precision for all samples. Between-day precision ranged between 0 and $2.5 \% \mathrm{CV}$ (Table 1). The mid-range sample of $552 \mu \mathrm{g} / \mathrm{l} \mathrm{FEU}$ showed a \%CV of $9.4 \% \mathrm{CV}$ for within-run and within-day precision and 2.5 $\% \mathrm{CV}$ for between-day precision.

\section{Healthy Reference Range}

Of the 762 replicates included in the health reference range study, 754 were valid samples. There was good agreement between test strip lots tested, and the overall healthy reference range threshold for the LumiraDx D-Dimer Test was calculated at a D-dimer value of $533 \mu \mathrm{g} / \mathrm{l}$ FEU, with $90 \%$ of results observed during this study being below this threshold (Table 2).

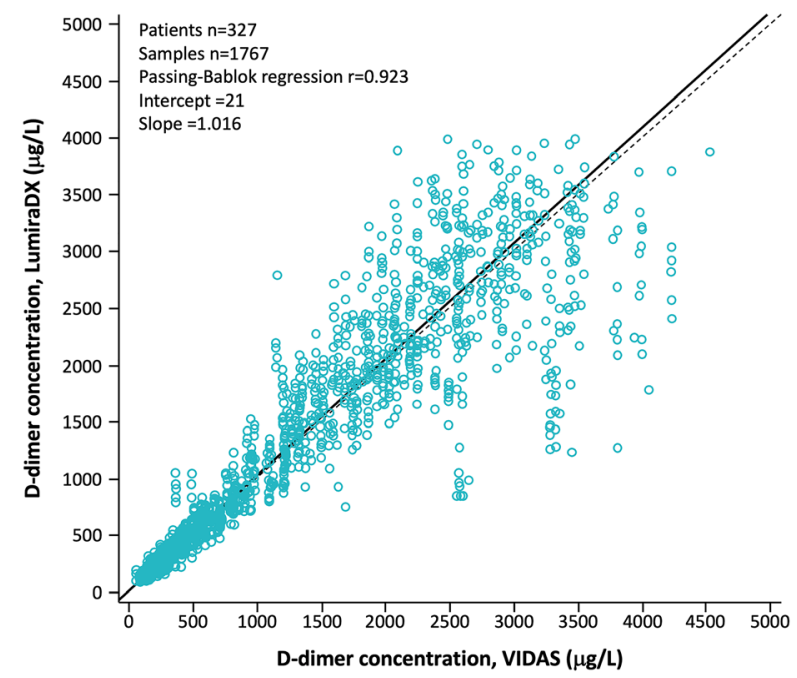

Fig. 1 Passing-Bablok regression scatter plot of D-dimer measurements from plasma samples obtained using the LumiraDx D-Dimer Test (LumiraDx) versus the bioMerieux VIDAS D-Dimer exclusion II immunoassay (VIDAS). The solid line represents the regression line and the dotted line the perfect fit with a slope of 1.0

Table 1 Precision levels of D-dimer concentrations in citrated venous plasma following a protocol based on CLSI EP5-A3

\begin{tabular}{llllll}
\hline $\begin{array}{l}\text { D-Dimer concentration } \\
(\boldsymbol{\mu g} / \mathbf{l ~ F E U})\end{array}$ & $\begin{array}{l}\text { Within-run } \\
\text { precision }(\% \mathbf{C V})\end{array}$ & $\begin{array}{l}\text { Within-day } \\
\text { precision }(\% \mathbf{C V})\end{array}$ & $\begin{array}{l}\text { Between-day } \\
\text { precision }(\% \mathbf{C V})\end{array}$ & $\begin{array}{l}\text { Total precision } \\
(\% \mathbf{C V})\end{array}$ & $\boldsymbol{n}$ \\
\hline 291 & 9.8 & 11.1 & 0.0 & 11.1 & 80 \\
552 & 9.4 & 9.4 & 2.5 & 9.7 & 80 \\
1790 & 10.1 & 10.1 & 0.7 & 10.2 & 80 \\
\hline
\end{tabular}

Three levels of D-dimer were tested in two runs of two replicates per day, for 20 days 

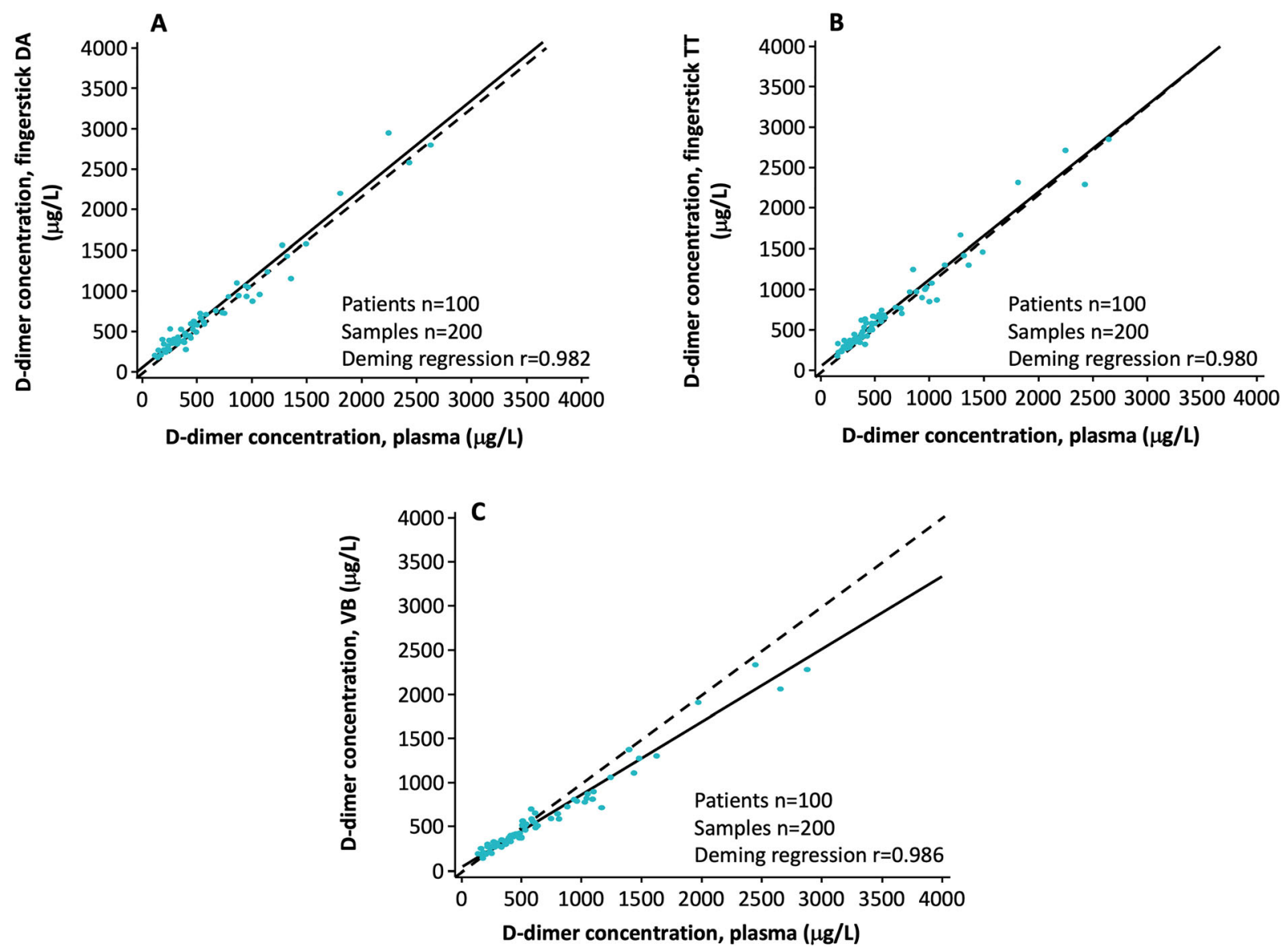

Fig. 2 Scatter plots for D-dimer levels analyzed using LumiraDx D-Dimer Test for alternative sample types compared to plasma samples from the same patient.

\section{Operator Usability at the POC}

Out of the 1101 total patient tests performed using the LumiraDx Diagnostic Platform, a use error by an operator resulting in a failed test occurred 51 times, a test alert occurred 14 times (hematocrit out of the $25-55 \%$ range), and an instrument error occurred one time (Table 3). These were error rates of $4.6,1.3$, and $0.09 \%$, respectively. The majority $(31 / 51)$ of the use errors were coded as 042 (insufficient sample) and this was corrected through a software update, taking the overall user error rate to $1.8 \%$ (Table 2).

The operator questionnaire evaluated metrics relating to usability and safety. Steps from setting up the machine to selecting the type of $r$ calculated using Deming regression for A fingerstick DA, B fingerstick TT and C VB DA, direct application; $T T$ transfer tube, $V B$ venous blood

-1) Strongly disagree $\| 2$ ) Disagree $=3$ ) Neutral $\square$ 4) Agree $\square$ 5) Strongly agree

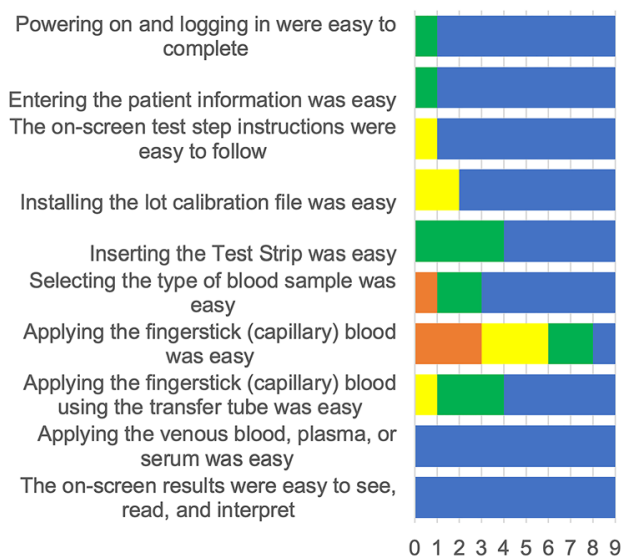

Fig. 3 Questionnaire responses. Usability responses from nine untrained test operators. Each question was completed using a five-point Likert scale, ranging from $1=$ strongly disagree to $5=$ strongly agree 
Table 2 Healthy reference range threshold by test strip lot

\begin{tabular}{llll}
\hline Strip lot & Range $(\mathbf{9 0 \%}$ cut-off) $(\boldsymbol{\mu g} / \mathbf{l})$ & Range $(\mathbf{9 5 \%}$ cut-off) $(\boldsymbol{\mu g} / \mathbf{l})$ & Range $(\mathbf{9 7 . 5 \%}$ cut-off) $(\boldsymbol{\mu g} / \mathbf{l})$ \\
\hline 5000055 & 486 & 636 & 1082 \\
5000056 & 547 & 712 & 1391 \\
5000059 & 552 & 707 & 1174 \\
Overall & 533 & 678 & 1082 \\
\hline
\end{tabular}

Table 3 Breakdown of the error codes observed in the precision study using the LumiraDx point-of-care device (1101 total tests, of which 468 were fingerstick tests plus 46 retests and 576 were VB tests plus 11 retests)

\begin{tabular}{lllc}
\hline Error code & Type of error & Description & Errors $(n)$ \\
\hline 002 & Use error & Door opened during test & 3 \\
004 & Use error & Sample applied too early & $3^{\mathrm{a}}$ \\
016 & Use error & Time exceeded to perform user action & 6 \\
038 & Use error & Insufficient sample or test timeout error & 8 \\
042 & Use error & Insufficient sample & 31 \\
019 & Test alert & Hematocrit out of range & 14 \\
2411 & Instrument error & Thresh check failure (a significant difference & 1 \\
& & in the measurement across the 3 test channels) &
\end{tabular}

a Includes 3503 error, which is a subset code for 004

blood sample were rated as easy by most respondents. One-third of operators $(3 / 9)$ did not agree that the direct application of the fingerstick sample was easy. However, they did successfully complete the tests and all operators agreed that applying the fingerstick using the transfer tube was straightforward. Similarly, most operators agreed that application of venous blood or plasma and the interpretation of results was uncomplicated (Fig. 3).

\section{DISCUSSION}

Method comparison of plasma samples using the LumiraDx D-Dimer Test versus the established laboratory-based bioMérieux VIDAS DDimer Exclusion II immunoassay demonstrated good equivalence between these two methods of analysis. Furthermore, the LumiraDx D-Dimer Test results were consistent across capillary fingerstick samples, venous whole blood, and plasma.

The method comparison of the LumiraDx DDimer Test versus the bioMérieux VIDAS DDimer Exclusion II immunoassay by Passing-Bablok regression and Pearson correlation met the performance criteria, with a regression for plasma samples $r=0.923$, and slope of 1.016. There was good agreement between fingerstick/VB samples and plasma samples $(r=0.980-0.986)$ measured using the LumiraDx D-Dimer Test. This indicates that the LumiraDx D-Dimer Test could replace current standard laboratory D-dimer quantifying methods in settings where laboratory facilities are not readily 
available, such as in primary care, without compromising analytical accuracy. The latest NICE guidance already recommends using quantitative POC testing within such settings, as part of a defined diagnostic pathway, which limits unnecessary referrals [8]. Previous studies using qualitative POC D-dimer assays have demonstrated substantial benefits in terms of reducing unnecessary referrals for imaging without increasing the number of patients with undiagnosed VTE [12]. A greater disparity between D-dimer measurements at concentrations $>1000 \mu \mathrm{g} / \mathrm{l}$ were observed between the LumiraDx D-Dimer Test and the bioMérieux VIDAS D-Dimer Exclusion II immunoassay (Fig. 1). Since the diagnostic pathway for VTE generally recommends using a D-dimer concentration $<500 \mu \mathrm{g} / \mathrm{l}$ to exclude VTE, correlation at the $<1000 \mu \mathrm{g} / \mathrm{l}$ is the most important parameter. Patients with elevated D-dimer levels require a diagnostic imaging test for definitive diagnosis [2]. Therefore, the authors are of the opinion that possible divergence between the measuring methods at these D-dimer concentrations would not impact the clinical utility of the D-dimer test. Moreover, D-dimer results vary between tests owing to the heterogeneity between D-dimer molecules and the difference between antibody specificity towards them. This is especially true concerning preference for high or low molecular weight degradation products and for cross-linked and non-crosslinked degradation products. This becomes more obvious at higher levels of D-dimer. It does not mean the test is not accurately detecting Ddimer, but that the correlation between the two different methods is weaker [23].

The healthy reference range determined a Ddimer threshold value of $533 \mu \mathrm{g} / \mathrm{l} \mathrm{FEU}$, which is in agreement with the generally accepted threshold of $500 \mu \mathrm{g} / 1[9,24]$. The fact that the LumiraDx D-Dimer Test is quantitative allows for its use at the point of care with fingerstick samples and results during the patient visit. Quantitation would also allow the straightforward implementation of age-adjusted D-dimer thresholds, as recommended by the latest NICE guidelines [8]. Although data are limited, the available literature suggests that the use of ageadjusted D-dimer thresholds is associated with increased diagnostic specificity compared with the use of unadjusted D-dimer thresholds (PE: 30\% [95\% confidence interval (CI): 19\%, 43\%] vs. $14 \%$ [95\% CI $8 \%, 25 \%]$; DVT: $44 \%$ [95\% CI $31 \%, 57 \%]$ vs. $27 \%$ [95\% CI $12 \%, 49 \%]$ ), and only a marginal decrease in sensitivity (PE: 96\% [95\% CI 94\%, 97\%] vs. 98\% [95\% CI 98\%, 99\%]; DVT: $91 \%$ [95\% CI 84\%, 96\%] vs. 96\% [95\% CI 89\%, 99\%]) [8].

While all results obtained at POC showed agreement and therefore equivalence with plasma results assessed in the laboratory, data for the VB suggested an under-recovery in high D-dimer results relative to the plasma results. However, the sample number at the higher end of the assay range was small, and the divergence was predominantly outside the clinical range. The assay showed consistent performance between plasma and capillary blood. The precision analyses showed a $\% \mathrm{CV}$ of under $10 \% \mathrm{CV}$ for the sample concentration of $552 \mu \mathrm{g} / \mathrm{l} \mathrm{FEU}$, indicating highest precision for this concentration range.

Usability testing initially indicated a common error (042, sample not detected) was reported most frequently. This error was resolved using a software update, resulting in a revised observed error rate of $1.8 \%$. Previously reported failure rates for qualitative and quantitative studies varied between 1 and $4 \%$ [25-27]. The failure rate for the quantitative POC test of $1.8 \%$ (corrected for the software update) falls within this range. Occasional user errors were noted, such as door closure too late, time out for sample application. Overall, the test was reported as easy to use by the operators. For those finding the direct application of sample difficult, the application of sample using a transfer tube represents a good alternative, as this was rated easy by most.

This performance evaluation has a number of limitations. Owing to the COVID-19 pandemic, the method comparison study was not able to prospectively recruit subjects and track clinical outcomes. Data were generated using plasma samples from a broad range of patients in the UK, USA, and Germany, with a range of clinical symptoms. In addition, a healthy reference population was studied to determine the 90th centile D-dimer level that would be 
considered elevated. Sufficient participant data were collected from a broad population to enable a statistically robust comparison of the LumiraDx D-Dimer Test with the reference bioMérieux VIDAS D-Dimer Exclusion II immunoassay. The prospective EMBOL-1 study is now in progress to determine the threshold for clinical rule out of VTE when used with the Wells Score (NCT NCT04737954). Furthermore, in order to compare sample matrices, venous, plasma, and capillary blood samples were collected and compared with the reference method in a US intended-use population. This product was evaluated under robust conditions to confirm its performance.

\section{CONCLUSIONS}

In conclusion, the quantitative LumiraDx DDimer Test was able to accurately measure Ddimer levels between 60 and $4515 \mu \mathrm{g} / \mathrm{l} \mathrm{FEU}$, with a low error rate for a range of blood sample types. When utilized in line with the latest diagnostic guidance [8], this technology could enable more accurate referrals for imaging diagnostics of VTE in settings such as primary care, where most patients initially present.

\section{ACKNOWLEDGEMENTS}

We thank the participants in this study, the clinical sites for sharing their space, and our research staff and nurses who conducted the study.

Funding. This performance evaluation was funded by LumiraDx. The study sponsor is also funding the journal's Rapid Service Fee.

Medical Writing Assistance. The authors acknowledge Kathrin Schulze-Schweifing of integrated medhealth communication (imc) for medical writing support, which was funded by LumiraDx.

Authorship. All named authors meet the International Committee of Medical Journal Editors (ICMJE) criteria for authorship for this article, take responsibility for the integrity of the work as a whole, and have given their approval for this version to be published.

Author Contributions. JE was responsible for the concept and design of the study, as well as drafting the manuscript. TJ generated the data and critically reviewed the manuscript. DC was responsible for the concept and design of the study, and critically reviewed the manuscript. AS, WS, and JK all contributed to the evaluation of the clinical data and critically reviewed the manuscript.

Disclosures. Jayne E Ellis, Thomas W Johnston, and David Craig are employees of LumiraDx. Anita Scribner, William Simon, and Judith Kirstein, declare that they have no conflicts of interest.

Compliance with Ethics Guidelines. The NOVEL clinical study received ethical approval from West of Scotland REC 3. NOVEL 3 (NCT04375982) was approved through WCG IRB (https://www.wcgirb.com/). Ethics approval for commercially obtained samples was obtained locally by the respective providers. All studies were performed in accordance with the Helsinki Declaration of 1964 and its later amendments and all participants provided informed consent prior to participation.

Data Availability. The datasets generated during and/or analyzed during the current study are available from the corresponding author on reasonable request.

Open Access. This article is licensed under a Creative Commons Attribution-NonCommercial 4.0 International License, which permits any non-commercial use, sharing, adaptation, distribution and reproduction in any medium or format, as long as you give appropriate credit to the original author(s) and the source, provide a link to the Creative Commons licence, and indicate if changes were made. The images or other third party material in this article are included in the article's Creative Commons licence, unless indicated otherwise in a credit line to the material. If material is not included in 
the article's Creative Commons licence and your intended use is not permitted by statutory regulation or exceeds the permitted use, you will need to obtain permission directly from the copyright holder. To view a copy of this licence, visit http:// creativecommons.org/licenses/by-nc/4.0/.

\section{REFERENCES}

1. Heit JA, Spencer FA, White RH. The epidemiology of venous thromboembolism. J Thromb Thromb. 2016;41(1):3-14

2. Weitz JI, Fredenburgh JC, Eikelboom JW. A test in context: D-dimer. J Am Coll Cardiol. 2017;70(19): 2411-20.

3. Heerink JS, et al. Analytical performance and userfriendliness of five novel point-of-care D-dimer assays. Scand J Clin Lab Invest. 2020;80(5):433-40.

4. Hendriksen JM, et al. Clinical characteristics associated with diagnostic delay of pulmonary embolism in primary care: a retrospective observational study. BMJ Open. 2017;7(3):e012789.

5. Walen S, et al. Diagnostic delay of pulmonary embolism in primary and secondary care: a retrospective cohort study. Br J Gen Pract. 2016;66(647): e444-50.

6. Verma K, Legnani C, Palareti G. Cost-minimization analysis of venous thromboembolism diagnosis: Comparison of standalone imaging with a strategy incorporating D-dimer for exclusion of venous thromboembolism. Res Pract Thromb Haemost. 2017;1(1):57-61.

7. Wells PS, et al. Evaluation of D-dimer in the diagnosis of suspected deep-vein thrombosis. N Engl J Med. 2003;349(13):1227-35.

8. National Institute for Health and Care Excellence. Venous thromboembolic diseases: diagnosis, management and thrombophilia testing NICE guideline [NG158]. 2020. https://www.nice.org.uk/guidance/ ng158/chapter/rationale-and-impact\#d-dimertesting-2. Accessed Apr 2021.

9. Wells PS, et al. Diagnosis of venous thromboembolism: 20 years of progress. Ann Intern Med. 2018;168(2):131-40.

10. Giannitsis E, et al. How to use D-Dimer in acute cardiovascular care. Eur Heart J Acute Cardiovasc Care. 2017;6(1):69-80.
11. Riley RS, et al. Widely used types and clinical applications of D-Dimer assay. Lab Med. 2016;47(2): 90-102.

12. Price CP, Fay M, Hopstaken RM. Point-of-Care Testing for D-Dimer in the Diagnosis of Venous Thromboembolism in Primary Care: A Narrative Review. Cardiol Ther. 2021;10(1):27-40.

13. All-Party Parliamentary Thrombosis Group. AllParty Parliamentary Thrombosis Group. DVT Diagnosis and Treatment in Primary Care. 2016; 2021. http://apptg.org.uk/wp-content/uploads/2016/12/ NHS-Innovation-Showcase.pdf. Accessed Apr 2021.

14. Buller HR, et al. Safely ruling out deep venous thrombosis in primary care. Ann Intern Med. 2009;150(4):229-35.

15. Geersing GJ, et al. Safe exclusion of pulmonary embolism using the Wells rule and qualitative Ddimer testing in primary care: prospective cohort study. BMJ. 2012;345:e6564.

16. Ten Cate-Hoek AJ, et al. Cost-effectiveness of ruling out deep venous thrombosis in primary care versus care as usual. J Thromb Haemost. 2009;7(12): 2042-9.

17. Emergency Medicine Research Group of Edinburgh. NOVEL Study. 2019. https://www.emergeresearch. org/trial/novel-study/. Accessed Apr 2021.

18. bioMérieux. VIDAS User manual. 2021. https:// www.biomerieux.co.uk/technical-library Accessed Jun 2021.

19. LumiraDx. LumiraDx. D-dimer test. 2021. https:// www.lumiradx.com/uk-en/what-we-do/ diagnostics/test-technology/d-dimer-test. Accessed Apr 2021.

20. CLSI. Measurement Procedure Comparisonand Bias Estimation Using Patient Samples. 3rd ed. CLSI guideline EP09c. Wayne, PA: Clinical and Laboratory Standards Institute; 20018.

21. CLSI. Defining, Establishing, and Verifying Reference Intervals in the Clinical Laboratory Approved Third edition; CSLI document EP28-A3c. Wayne, PA: Clinical and Laboratory Standards Institute; 2008.

22. CLSI. Evaluation of precision of quantiative measurement procedures; Approved Guideline - Third Edition. CLSI document EP05-A3. Wayne, PA: Clinical and Laboratory Standards Institute; 2014.

23. Kogan AE, et al. Monoclonal antibodies with equal specificity to D-dimer and high-molecular-weight fibrin degradation products. Blood Coagul Fibrinolysis. 2016;27(5):542-50. 
24. BioMerieux. VIDAS ${ }^{\circledR}$ D DIMER EXCLUSION II product information. 22/01/2021; 2021. https:// www.mybiomerieux.com/en_US/

cleverlink? productnumber=30455-01. Accessed Apr 2021.

25. Lucassen WA, et al. Qualitative point-of-care Ddimer testing compared with quantitative D-dimer testing in excluding pulmonary embolism in primary care. J Thromb Haemost. 2015;13(6):1004-9.
26. van Belle A, et al. Effectiveness of managing suspected pulmonary embolism using an algorithm combining clinical probability, D-dimer testing, and computed tomography. JAMA. 2006;295(2):172-9.

27. Perrier A, et al. Multidetector-row computed tomography in suspected pulmonary embolism. N Engl J Med. 2005;352(17):1760-8. 